\title{
Modelo de formación del profesorado en tecnología educativa. ${ }^{1}$ \\ Training Model on Educational Technology for Teachers.
}

\author{
Glenn Elmer Hernández Camelo² \\ ${ }^{1}$ Universidad de Granada, España.
}

Artículo recibido en el mes de Marzo de 2014; artículo aceptado en el mes de Junio de 2014

Citación del artículo: Hernández, G. (2014). Modelo de formación del profesorado en tecnología educativa. I+D Revista de Investigaciones, 3(1), 18-23.

\section{Resumen}

Se presentan los resultados parciales del estudio sobre un modelo de formación del profesorado, para abordar el proceso de enseñanza mediante la tecnología educativa en el municipio de San Juan Girón. Se llevó a cabo un estudio no experimental, descriptivo, transversal. Se describen las competencias en tecnologías de la información y la comunicación, y su aplicación por parte de los docentes de los colegios del municipio; también la percepción que ellos, los directivos de éstos y los estudiantes, tienen sobre la aplicación de las tecnologías de la información y la comunicación en el aula.

Palabras clave: Competencias, educación, formación docente, metodología, tecnología educativa.

\section{Abstract.}

This study shows the partial cut results about a training model on educational technology for teachers to develop a set of skills for their applying it in the teaching-learning process at secondary school in the municipality of San Juan Girón (Colombia). A non-experimental-crossdescriptive investigation was carried out. Information and Communications Technology skills to be developed in teachers at the secondary level of education are described, as well as the way they should apply them in teaching; the educator's, managing staff's and

\footnotetext{
${ }^{1}$ Artículo de investigación científica, de enfoque cuantitativo, resultado parcial de un proyecto de investigación en curso denominado: Propuesta de un modelo de formación para la enseñanza mediante tecnología educativa, desarrollado en el grupo de investigación AREA (Análisis de la Realidad Educativa Andaluza) y fue financiado por la Universidad de Granada (España). Dirección: Avda. del Hospicio, C.P. 18071 Granada - Tel: (34)958243000. Correo electrónico: informa@ugr.es

${ }^{2}$ Ingeniero Electrónico, Universidad del Valle. Magíster en Ingeniería, Área Telecomunicaciones, Universidad Pontificia Bolivariana. Docente-Investigador del grupo: AREA. Universidad de Granada (España). Correo electrónico: glenn.hernandezc@gmail.com
} 
GLENN ELMER HERNÁNDEZ CAMELO

Modelo de formación del profesorado en tecnología educativa.

student's perception about their use in the classroom is examined.

Keywords: Skill, education, teacher training, methodology, educational technology.

\section{Introducción}

La creación de nuevos escenarios y entornos más ricos y variados para el aprendizaje, adaptados a las nuevas demandas y exigencias de los nuevos retos educativos, ha demandado la inclusión de las tecnologías de la información y la comunicación en los procesos educativos y con ello ha exigido a los docentes hacer uso de ellas y prepararse para su utilización en el aula (Cabero, 2004; Sánchez \& Lombardo, 2004); más allá de esto, ha demandado en él un rol más participativo y generativo, como productor y/o adaptador de los medios tecnológicos a las necesidades y características de los estudiantes, a sus demandas cognitivas y estilos de aprendizaje (Cabrero, 2005). De esta forma, la integración de las tecnologías de la información en el currículo, se plantea como un mecanismo de desarrollo del aprendizaje mismo, no solo como objeto en sí mismo, sino como medio para que éste se realice, es decir, que integre el modelo pedagógico (Aguaded, 1993); es por ello que al hablar de TIC en la educación, involucra no solamente la adquisición de equipos de cómputo y herramientas ofimáticas, sino de un análisis de contenidos, competencias, de habilidades docentes que permitan abordar esta nueva estrategia de aprendizaje y metodología de una manera eficaz, específicamente mediante el uso de herramientas Web 2.0. En este sentido, se espera que el centro de atención sigan siendo las estrategias, metodologías y competencias que se deben desarrollar en los estudiantes a través de la aplicación de las TIC, y no la adaptación de los procesos educativos a los adelantos tecnológicos que se encuentren en uso en un determinado momento (Mendoza \& Michalay, 2004); de allí que la capacitación de los profesores debe implicar los elementos de apoyo que les facilitan las TIC (Buzón, 2005). Esta capacitación va dirigida a competencias muy específicas y bajo estándares definidos (UNESCO, 2008), dirigidas a ser un orientador, facilitador de conocimientos y elaborador de diversos tipos de materiales multimedia, generando así nuevas oportunidades de aprendizaje apoyadas en las TIC.

Con base en lo anterior, el presente estudio gira en torno a la pregunta acerca de ¿Qué variables, estructura, contenidos, funciones, deben constituir un modelo de formación del profesorado en tecnología educativa en instituciones de educación en el municipio de San Juan Girón?.

\section{Método}

\section{Tipo de estudio}

Se siguió un tipo de investigación no experimental, descriptiva transversal.

\section{Participantes}

La población objetivo de la investigación son las Instituciones de Educación (Básica y Media) del municipio de San Juan Girón en el departamento de Santander (Colombia). Actualmente existen cincuenta instituciones de educación, de las cuales el 30\% (15 instituciones) son de carácter público y el 70\% 
(35 instituciones) son de carácter privado. El municipio de San Juan Girón cuenta con 29.081 estudiantes, de los cuales el 75.64\% (21.999 estudiantes) se encuentra en el sector público y el $24.35 \%$ (7.082 estudiantes) en el sector privado. El número total de docentes es de 746 . El número de directores de los centros educativos se eleva a 50 , de los cuales el 30\% (15 directores) son del sector público y el 70\% (35 directores) son del sector privado. Actualmente existen 40 coordinadores de instituciones educativas que se encuentran en el sector público. La población total del municipio de San Juan Girón es de 136.514 personas, de las cuales el $86.91 \%$ (118.665 habitantes) se encuentra ubicado en la cabecera municipal y el $13.08 \%$ (17.859 habitantes) se encuentran ubicados en zona rural. De acuerdo con lo anterior, y teniendo en cuenta las características de la investigación, se seleccionaron 836 participantes correspondientes al personal docente, directores de instituciones de educación y coordinadores de las mismas. De estos, se tomó el $40 \%$ como porcentaje significativo, logrando así tener una muestra de 334 habitantes correspondientes al $40 \%$ de los docentes (298,4 docentes), al 40\% de los directores de instituciones (20 directores) y al $40 \%$ de los coordinadores ( 16 coordinadores).

\section{Materiales e instrumentos cuestionario.}

Se aplicó un tipo de cuestionario a tres poblaciones diferentes, pero que guardan relación con el objeto de estudio propuesto. Los cuestionarios están orientados hacia los Directores de las Instituciones de Educación, los Coordinadores de las mismas y finalmente a los Docentes pertenecientes a estas instituciones.

\section{Procedimiento}

Se siguió un análisis de modelamiento mediante simulación de variables que influyen en un sistema, que ha permitido estimar el comportamiento de las mismas con el propósito de establecer acciones de mejora. En el caso educativo, la aplicación y utilización de herramientas estadísticas ha permitido establecer el comportamiento de diferentes variables, que por su naturaleza y magnitud resulta difícil estimar. Además, el uso de herramientas estadísticas como el SPSS ${ }^{\circledR}$ y Statgraphics $\AA$, permite la realización de simulaciones con el propósito de establecer la respuesta del sistema ante la manipulación de diferentes valores de una variable, identificando así, el grado de afectación que tiene la misma en el estudio realizado. Además, estos software permiten también la aplicación de modelos estadísticos que ayudan a determinar el comportamiento de las variables, con el propósito de conocer si siguen un patrón establecido. Con relación propuesta de investigación, la aplicación del software permitirá establecer las características que tienen todos los directores, coordinadores y docentes estudiados, en relación con las variables seleccionadas, logrando así establecer el grado de incidencia que han tenido en el proceso y cómo a través de la manipulación o variación de las mismas, se pueden obtener mejores resultados que se verán reflejados en el cumplimiento de los objetivos y por ende en la calidad educativa. 
GLENN ELMER HERNÁNDEZ CAMELO

Modelo de formación del profesorado en tecnología educativa.

\section{Resultados}

Se presentan los resultados preliminares del estudio, a través de la recopilación de datos de las encuestas realizadas.

El $50 \%$ de las instituciones encuestadas respondieron que el porcentaje de directivos docentes y docentes formados en el desarrollo de planes de gestión con el uso de TIC, está entre el $1 \%$ y $25 \%$.

El $75 \%$ de las instituciones encuestadas respondieron que el porcentaje de Docentes formados en el momento de apropiación profesional en uso de TIC, está entre el 1\% y $25 \%$.

El $50 \%$ de las instituciones educativas encuestadas sobre programas de formación de

22 directivos y docentes en el uso educativo de TIC, respondieron que se desarrollan programas de formación en TIC sin diagnóstico para detectar necesidades de capacitación.

El 100\% respondió que no se desarrollan programas de formación en TIC a partir de la identificación de necesidades de formación, y no existe acompañamiento y seguimiento.

El 100\% respondió que no se desarrollan programas de formación en TIC con necesidades, estrategias, acompañamiento y seguimiento.

El $50 \%$ contestó que menos del $25 \%$ de los programas de formación que realiza la Secretaría de Educación, incluyen el uso de medios y TIC.
El $75 \%$ contestó que no se han planteado acciones que fortalezcan el acceso a la información a través de la generación de contenidos educativos o a través de la adquisición a terceros.

El $12.5 \%$ respondió que existen estrategias para fomentar el uso de los contenidos generados por los docentes de los establecimientos educativos.

El 87\% respondió que los establecimientos educativos que cuentan con estrategias de producción y gestión de contenidos educativos a través de internet, radio y televisión, están entre el $1 \%$ y el $25 \%$. El resto (12.5\%) se encuentra en $0 \%$.

E1 50\% de las instituciones encuestadas tienen un porcentaje de participación de docentes en redes y comunidades de aprendizaje entre un $1 \%$ y un $25 \%$.

En el $75 \%$ de las instituciones, no se tienen estrategias de fomento al uso pedagógico de herramientas Web 2.0 por parte de los docentes y estudiantes.

E1 75\% de las instituciones contestaron que la Secretaría de Educación no tiene estrategias de fomento de comunidades de aprendizaje.

El $87.5 \%$ de las instituciones respondieron que no existen estrategias que fomenten el uso pedagógico de TIC en las personas de diferentes grupos étnicos o diversidad cultural.

El $87.5 \%$ de las instituciones respondieron que no existen estrategias que fomenten el uso 
pedagógico de TIC en las personas con necesidades especiales.

E1 50\% de las instituciones contestaron que no se realiza acompañamiento ni seguimiento a las estrategias institucionales de uso pedagógico de medios y TIC de los Establecimientos Educativos.

E1 50\% de las instituciones respondieron que no se ha contemplado el uso de TIC para fortalecer de forma transversal las diferentes áreas del conocimiento que se desarrollan en los Establecimientos Educativos.

\section{Discusión}

Los resultados preliminares obtenidos, no permiten aún hacer discusiones sustentadas; sin embargo, preliminarmente se obtiene que el escenario para el manejo y capacitación del profesorado en las TIC, presenta carencias significativas que derivan en la inclusión de las mismas en los planes y programas didácticos en las instituciones.

\section{Referencias}

Aguaded, I. (1993). Comunicación audiovisual en una enseñanza renovada: Propuestas desde los medios. Recuperado de h t t p s : / / m a n a r e a . w e b s . u 11. es / w p content/uploads/2010/06/ebookte.pdf

Buzón García, O. (2005). La incorporación de plataformas virtuales a la enseñanza: una experiencia de formación on-line basada en competencias. Fundación Dialnet, 4(1), 77-100.

Cabero, J. (2005). Estrategias para la formación del profesorado en TIC. Universidad de Sevilla. España. Recuperado a partir de http://www.ciedhumano.org/files/Edutec2005 j ULIO.pdf

Cabero, J. (2004). Formación del Profesorado en TIC. En II Congreso Nacional de Formación de Profesorado en TIC. Jaén, España: Universidad de Jaén. Recuperado de http://www.ujaen.es/huesped/forprofe/program a.html

Mendoza, J., \& Milachay, V. (2004). TICS en la formación inicial y permanente del profesorado educación especial: Universidad de Costa Rica. E-Prints Complutense. Universidad de Costa Rica. Recuperado de h t t p : / / a g m . c a t / r e c e r c a divulgacio/UsoTIC_formacionProf_DCES.pdf

Sánchez García, L., \& Lombardo, J. M. (2004). Las TIC y la formación del profesorado en la Enseñanza Secundaria. Revista Educación y Futuro, 3(1),1-1.

UNESCO. (2008). Normas UNESCO sobre Competencias en TIC para Docentes. Organización de Estados Iberoamericanos. Recuperado de http://www.oei.es/index.php 